EPJ Web of Conferences 21, 03001 (2012)

DOI: $10.1051 /$ epjconf/20122103001

(C) Owned by the authors, published by EDP Sciences, 2012

\title{
PRESENT STATUS AND FUTURE PROGRAMS OF THE n_TOF EXPERIMENT
}

E. Chiaveri ${ }^{1)}$, M. Calviani ${ }^{1)}$, V. Vlachoudis ${ }^{1)}$, C. Weiß ${ }^{2)}$, S. Andriamonje ${ }^{1)}$, J. Andrzejewski ${ }^{3)}$,

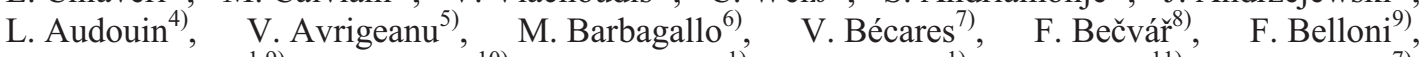
E. Berthoumieux $^{1,9)}$, J. Billowes ${ }^{10)}$, V. Boccone ${ }^{1)}$, M. Brugger ${ }^{1)}$, F. Calviño ${ }^{11)}$, D. Cano-Ott ${ }^{7)}$, C. Carrapiçoco ${ }^{12)}$, F. Cerutti ${ }^{1)}$, M. Chin ${ }^{1)}$, N. Colonna ${ }^{6}$, G. Cortés ${ }^{11)}$, M.A. Cortés-Giraldo ${ }^{13)}$, M. Diakaki ${ }^{14)}$, I. Dillmann ${ }^{15)}$, C. Domingo-Pardo ${ }^{16)}$, I. Duran ${ }^{17)}$, N. Dzysiuk ${ }^{18)}$, C. Eleftheriadis ${ }^{19)}$, M. Fernández-Ordóñez ${ }^{7)}$, A. Ferrari ${ }^{1)}$, K. Fraval ${ }^{9)}$, S. Ganesan ${ }^{20)}$, G. Giubrone ${ }^{21)}$, M.B. GómezHornillos $^{11)}$, I.F. Gonçalves ${ }^{12)}$, E. González-Romero ${ }^{7)}$ F. Gramegna ${ }^{18)}$, E. Griesmayer ${ }^{2)}$, C. Guerrero ${ }^{1)}$, F. Gunsing ${ }^{9}$, M. Heil ${ }^{16)}$, D.G. Jenkins ${ }^{22)}$, E. Jericha ${ }^{2)}$, Y. Kadi ${ }^{1)}$, F. Käppeler ${ }^{23)}$, D. Karadimos ${ }^{24)}$, M. Kokkoris ${ }^{14)}$, M. Krtička ${ }^{8)}$, J. Kroll ${ }^{8)}$, C. Lederer ${ }^{25)}$, H. Leeb ${ }^{2)}$, L.S. Leong), R. Losito ${ }^{1)}$, M. Lozano ${ }^{13)}$, A. Manousos ${ }^{19)}$, J. Marganiec ${ }^{3)}$, T. Martinez ${ }^{7)}$, C. Massimi ${ }^{26)}$, P.F. Mastinu ${ }^{18)}$, M. Mastromarco ${ }^{6)}$, M. Meaze ${ }^{6)}$, E. Mendoza ${ }^{7)}$, A. Mengoni2 ${ }^{27)}$, P.M. Milazzo ${ }^{28)}$,

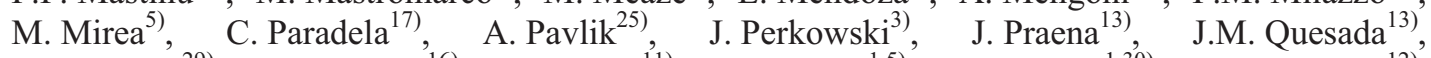
T. Rauscher ${ }^{29)}$, R. Reifarth ${ }^{16)}$ A. Riego ${ }^{11)}$, F. Roman ${ }^{1,5)}$, C. Rubbia ${ }^{1,30)}$, R. Sarmento ${ }^{12)}$, G. Tagliente $^{6)}$, J.L. Tain ${ }^{21)}$, D. Tarrìo ${ }^{17)}$, L. Tassan-Got ${ }^{4)}$, A. Tsinganis ${ }^{1)}$, S. Valenta ${ }^{8)}$, G. Vannini ${ }^{26)}$,

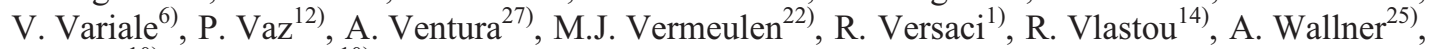
T. Ware ${ }^{10)}$, T.J. Wright ${ }^{10)}$

1) European Organization for Nuclear Research (CERN), Geneva, Switzerland

2) Atominstitut, Technische Universität Wien, Austria

3) Uniwersytet Łódzki, Lodz, Poland

4) Centre National de la Recherche Scientifique/IN2P3 - IPN, Orsay, France

5) Horia Hulubei National Institute of Physics and Nuclear Engineering - IFIN HH, Bucharest Magurele, Romania

6) Istituto Nazionale di Fisica Nucleare, Bari, Italy

7) Centro de Investigaciones Energeticas Medioambientales y Technologicas (CIEMAT), Madrid, Spain

8) Charles University, Prague, Czech Republic

9) Commissariat à l'Énergie Atomique (CEA) Saclay - Irfu, Gif-sur-Yvette, France

10) University of Manchester, Oxford Road, Manchester, UK

11) Universitat Politecnica de Catalunya, Barcelona, Spain

12) Instituto Tecnológico e Nuclear (ITN), Lisbon, Portugal

13) Universidad de Sevilla, Spain

14) National Technical University of Athens (NTUA), Greece

15) Physik Department E12 and Excellence Cluster Universe, Technische Universität München, Munich, Germany

16) GSI Helmholtzzentrum für Schwerionenforschung GmbH, Darmstadt, Germany

17) Universidade de Santiago de Compostela, Spain

18) Istituto Nazionale di Fisica Nucleare, Laboratori Nazionali di Legnaro, Italy

19) Aristotle University of Thessaloniki, Thessaloniki, Greece

20) Bhabha Atomic Research Centre (BARC), Mumbai, India

21) Instituto de Fìsica Corpuscular, CSIC-Universidad de Valencia, Spain

22) University of York, Heslington, York, UK

23) Karlsruhe Institute of Technology, Campus Nord, Institut für Kernphysik, Karlsruhe, Germany

24) University of Ioannina, Greece

25) University of Vienna, Faculty of Physics, Austria

26) Dipartimento di Fisica, Università di Bologna, and Sezione INFN di Bologna, Italy

This is an Open Access article distributed under the terms of the Creative Commons Attribution-Noncommercial License 3.0, which permits unrestricted use, distribution, and reproduction in any noncommercial medium, provided the original work is properly cited. 
27) Agenzia nazionale per le nuove tecnologie, l'energia e lo sviluppo economico sostenibile (ENEA), Bologna, Italy

28) Istituto Nazionale di Fisica Nucleare, Trieste, Italy

29) Department of Physics and Astronomy - University of Basel, Basel, Switzerland

30) Laboratori Nazionali del Gran Sasso dell'INFN, Assergi (AQ),Italy

The neutron time-of-flight facility n_TOF at CERN, Switzerland, operational since 2001, delivers neutrons using the Proton Synchrotron (PS) $20 \mathrm{GeV} / \mathrm{c}$ proton beam impinging on a lead spallation target. The facility combines a very high instantaneous neutron flux, an excellent time of flight resolution due to the distance between the experimental area and the production target (185 meters), a low intrinsic background and a wide range of neutron energies, from thermal to GeV neutrons.

These characteristics provide a unique possibility to perform neutron-induced capture and fission cross-section measurements for applications in nuclear astrophysics and in nuclear reactor technology.

The most relevant measurements performed up to now and foreseen for the future will be presented in this contribution. The overall efficiency of the experimental program and the range of possible measurements achievable with the construction of a second experimental area (EAR-2), vertically located $20 \mathrm{~m}$ on top of the n_TOF spallation target, might offer a substantial improvement in measurement sensitivities. A feasibility study of the possible realisation of the installation extension will be also presented.

\section{INTRODUCTION}

High precision neutron cross-section data are of major importance for a wide variety of research fields in basic and applied nuclear physics [1]. In particular, neutron data on neutron-nucleus reactions are essential in Nuclear Astrophysics for understanding the production rate of heavy elements in the Universe, which occurs mainly through slow and rapid neutron capture processes during the various phases of stellar evolution [2,3]. In the field of nuclear technology new studies aimed at developing future generation nuclear system which would address major safety, proliferation and waste concerns are requiring more accurate data in neutron-induced fission and capture cross-section on radioactive isotopes. Based on these motivations the neutron time-of-flight facility n_TOF has been proposed at CERN, Geneva

\section{II. n_TOF FACILITY}

The idea of a new neutron time-of-flight facility at CERN was proposed by C. Rubbia in 1998 [4], as a follow up of the TARC experiment, also conducted at CERN. 


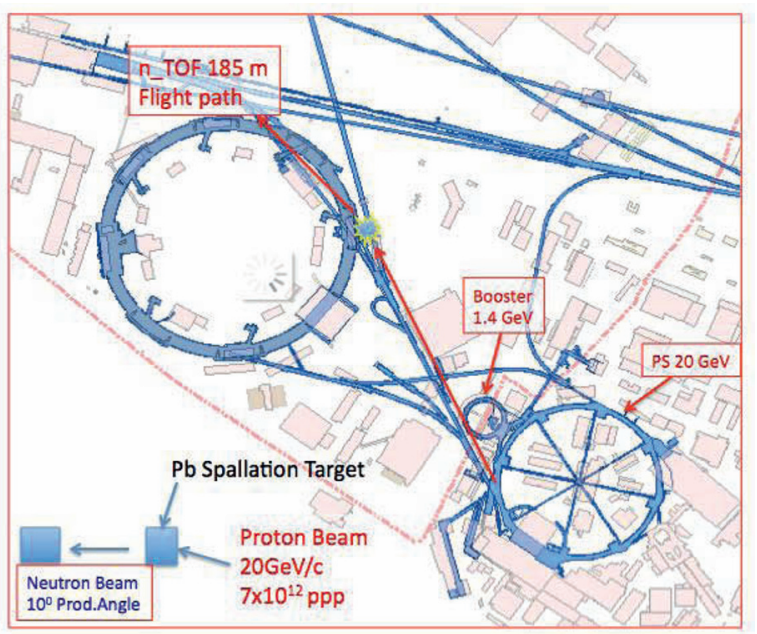

Figure 1: Schematic view of the proton acceleration complex for $n$ _TOF Facility

The concept of the n_TOF neutron beam [5] makes use of both the specifically high flux of neutrons attainable using the spallation process of $20 \mathrm{GeV} / \mathrm{c}$ protons on a massive lead target (see Fig.1), able to contain practically the whole spallation shower as well as the remarkable beam density of the CERN Proton Synchrotron (PS) [6]. After the initial proposal, in a short amount of time the facility was accepted for construction by CERN. The CERN n_TOF facility has been set in operation and commissioned in 2001 with performances matching the expectations. The PS machine of CERN can generate high intensities up to $7 \times 10^{12}$ ppp (protons per pulse) - high enough to produce the vast number of $2 \times 10^{15}$ neutrons per pulse - in the form of short ( 6 ns width) pulses with a repetition time varying from $1.2 \mathrm{~s}$ to $16.7 \mathrm{~s}$ and a prompt "flash" considerably smaller compared to electron machines. The high neutron flux, the low repetition rates and the excellent energy resolution of $5.5 \times 10^{-4}(1 \mathrm{KeV})$ have opened new possibilities for high precision cross section measurements in the energy range from thermal to $\mathrm{GeV}$, for stable and, in particular, for radioactive targets. During the first years of operation 2001-2004 the n_TOF collaboration has attained a rich experimental program measuring in total 36 isotopes and producing numerous scientific papers and proceedings. (see table1 ) [7-25].

\begin{tabular}{|c|c|}
\hline $\begin{array}{l}\text { Capture } \\
{ }^{151} \mathrm{Sm}\end{array}$ & Measurement campaign 2002-4 \\
\hline $204,206,207,208 \mathrm{~Pb},{ }^{209} \mathrm{Bi}$ & \\
\hline $24,25,26 \mathrm{Mg}$ & - Measurements of capture reactions: \\
\hline $90,91,92,94,96 \mathrm{Zr},{ }^{93} \mathrm{Zr}$ & - 25 Isotopes (8 of which radioactive) \\
\hline${ }^{186,187,188} \mathrm{Os}$, ${ }^{139} \mathrm{La}$ & - Often of double interest (Astrophysics and applications) \\
\hline${ }^{232} \mathrm{Th}, 233,234 \mathrm{U}$ & - Most results already available \\
\hline${ }^{237} \mathrm{~Np},{ }^{240} \mathrm{Pu},{ }^{243} \mathrm{Am}$ & - Several publications \\
\hline Fission & - Measurements of fission cross-sections: \\
\hline $233,234,235,236,238 \mathrm{U}$ & - 11 isotopes (10 radioactive) \\
\hline${ }^{232} \mathrm{Th},{ }^{209} \mathrm{Bi}$ & - strong interest to the data bv International Nuclear Agencies \\
\hline${ }^{237} \mathrm{~Np}$ & - Results are now becoming available (PRCs) \\
\hline${ }^{241,243} \mathrm{Am},{ }^{245} \mathrm{Cm}$ & \\
\hline
\end{tabular}

Table 1: List of performed measurements of capture and fission cross-sections at $\mathrm{n}$ TOF. In the first column on the left side the blue marked isotopes are stable while the red one are radioactive. 


\section{III. n TOF FACILITY FROM 2008}

After three years long stop due to cooling water activation by spallation products diluted in the water, a new lead target was constructed, and new systems developed in order to meet safety requirements for the restart of the facility. In particular, a ventilation system was installed in the primary target area, while a new optimized cooling system was developed, which allows to control and balance the chemical parameters of the water (in particular the oxygen content and conductivity) and to properly filter activation products eventually present in the water circuit. An extensive study of target corrosion mechanism had been performed, in order to improve the longterm stability of the system. A new cylindrical shape lead block of $40 \mathrm{~cm}$ radius and $60 \mathrm{~cm}$ length, developed in order to optimize cooling and neutron production was designed and built. The new target assembly consists of a separated cooling and moderator circuit, which enables the use of different moderator materials, thus allowing a greater flexibility on the characteristics of the neutron beam.

After the commissioning in which started in November 2008 and continue during mid-2009 two other changes have been implemented; the first is the implementation of a different moderator material other than light water. In particular, the program has envisaged the use of water enriched in ${ }^{10} \mathrm{~B}$, which has greatly enhanced the measuring capabilities of neutron-induced capture crosssection, by reducing significantly the presence of the in-beam photon component, and in particular the $2.2 \mathrm{MeV} \gamma$-rays, produced both by neutron capture in hydrogen. Since this photon contribution is delayed, i.e. emitted after about 1 microsecond from the proton interaction (due to the fact that it is produced by thermalized neutrons), it would results in a background component in the $1-100 \mathrm{keV}$ neutron energy range, which is problematic for capture reaction measurements performed with C6D6 detectors. The conceived system has reduced the $2.2 \mathrm{MeV}$ photon components by a factor of about 10, leaving the neutron fluence unchanged above $1 \mathrm{eV}$.

Another upgrade performed during the 2010 run has been the transformation of the $\mathrm{n}$-TOF experimental area into a Work Sector Type A, which has allowed the possibility to perform measurements of capture and fission cross-section of "unsealed" samples of highly radioactive isotopes, such as actinides like ${ }^{241} \mathrm{Am},{ }^{243} \mathrm{Am}$ and ${ }^{240-2} \mathrm{Pu}$, taking full advantage of the facility's high instantaneous neutron flux. This has required a complete revision of the experimental area and of the related technical services. The milestone of $\mathrm{n}_{-}$TOF facility is summing-up on fig. 2

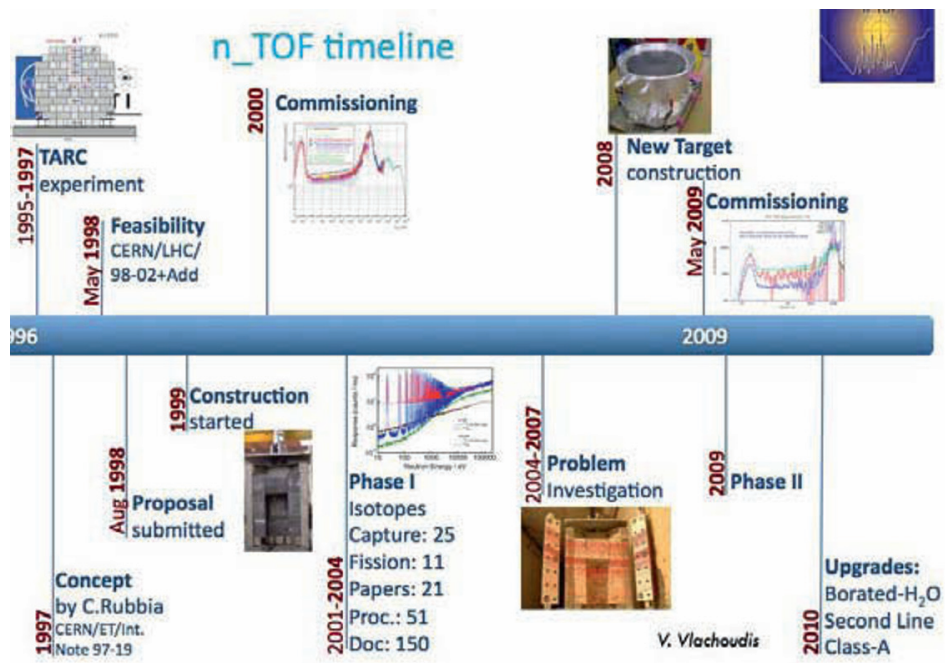

Figure 2: Milestone of n_TOF Facility 


\section{OVERVIEW OF NOTABLE RESULTS ON CAPTURE AND FISSION CROSS SECTION}

Among the measurements presented on the table 1, some additional details will be given here, as they represent significant results obtained at $\mathrm{n} \_$TOF experiment.

The data on neutron-induced fission cross section of minor actinides - and in particular for $\mathrm{Cm}$ and Am isotopes - are scarce and show large discrepancies between the available measurements: for these reasons, in 2004 a campaign has started on the measurement of the fission cross-section of these isotopes, using a Fast Ionization Chamber (FIC) specifically built for n_TOF.

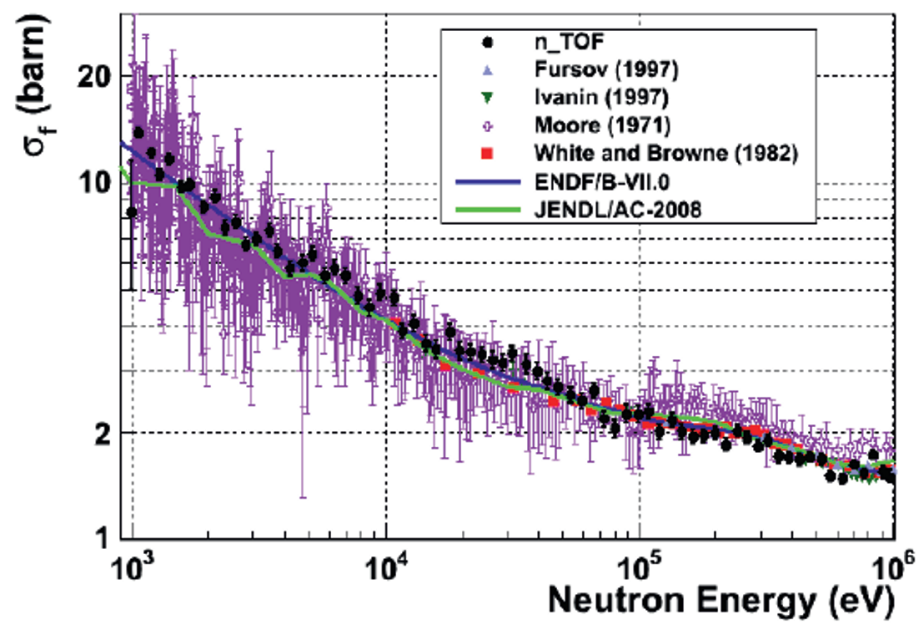

Figure $3{ }^{245} \mathrm{Cm}(n, f) n$ TOF data between $1 \mathrm{keV}$ and $1 \mathrm{MeV}$, compared with evaluated libraries and previous experimental data (metti referenza del mio articolo ND2010).

Figure 3 shows the ${ }^{245} \mathrm{Cm}(\mathrm{n}, \mathrm{f}) \mathrm{n} \_\mathrm{TOF}$ data results between $1 \mathrm{keV}$ and $1 \mathrm{MeV}$ compared with evaluated libraries and previous measurement: in this energy range the n_TOF data are in average within 2\% from ENDF/B-VII.0 and 3\% from Moore et al.. The systematic uncertainty of the $\mathrm{n} \_$TOF data, including the value induced by the normalization, accounts to about $5 \%$ in the full neutron energy range.[26]

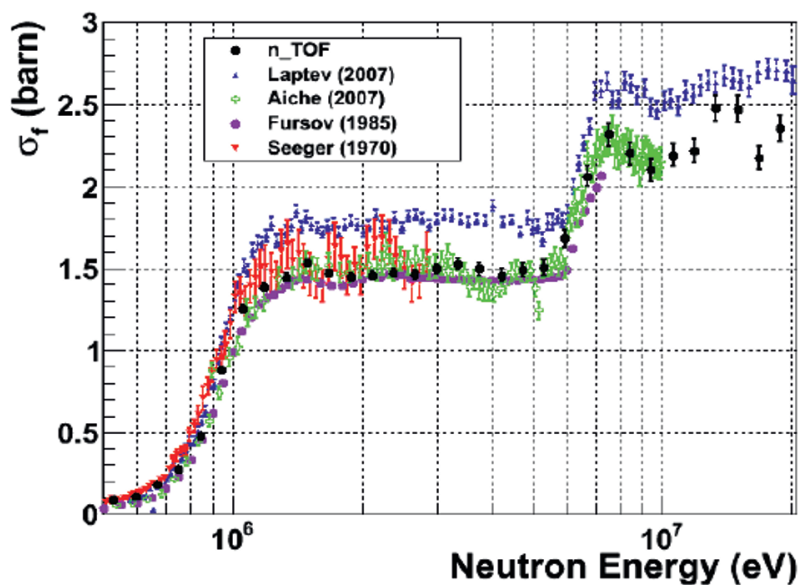

Figure 4. 243Am(n,f) cross-section measured at $\hat{n}$ TOF around the fission threshold, compared with ENDF/B-VII.0 and previous experimental results. 
Figure 4 shows the $n_{-}$TOF data on the ${ }^{243} \mathrm{Am}(\mathrm{n}, \mathrm{f})$ cross-section from $500 \mathrm{keV}$ to $20 \mathrm{MeV}$ compared to ENDF/B-VII.0 and previous results. The experimental points clearly show that the recent data by Laptev et al. overestimate the cross-section, while confirming the evaluations of ENDF/B-VII.0.[26]

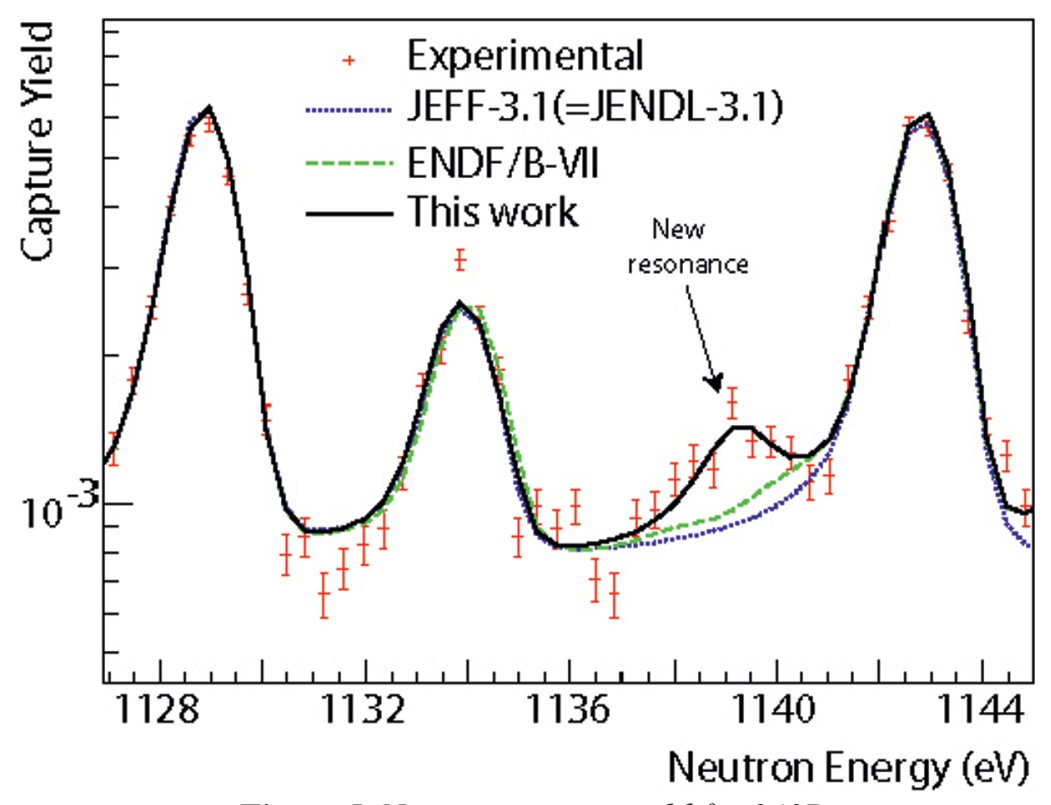

Figure 5. Neutron capture yield for 240Pu.

Figure 5 shows an example of the capture yield on actinides measured at $n$ _TOF: the $n$ TOF data (red points) are ${ }^{240} \mathrm{Pu}(\mathrm{n} . \mathrm{g})$ fission yield analyzed with the SAMMY code (black line) and compared to the JEFF3.1 evaluated database. The high resolving power at the $185 \mathrm{~m}$ flight path has allowed identifying 6 new resonances in the $\mathrm{keV}$ region [27].

Another example of the $\mathrm{n}$-TOF measurement capabilities and of the vast experimental campaign is given by the measurement of the capture cross-section of $186 \mathrm{Os}$ and 187Os, which has resulted also in a series of featured articles on Phys. Rev. C (http://physics.aps.org/synopsisfor/10.1103/PhysRevC.82.015802).

The precise determination of the neutron capture cross sections of these two isotopes is important to define the $s$-process abundance of $187 \mathrm{Os}$ at the formation of the solar system. This quantity can be used to evaluate the radiogenic component of the abundance of $187 \mathrm{Os}$ due to the decay of the unstable $187 \operatorname{Re}(t 1 / 2=41.2 \mathrm{Gyr})$ and from this to infer the time duration of the nucleosynthesis in our galaxy (Re/Os cosmochronometer). The neutron capture cross sections of $186 \mathrm{Os}, 187 \mathrm{Os}$, and 1880s have been measured at the CERN n TOF facility from $1 \mathrm{eV}$ to $1 \mathrm{MeV}$ (see for example Fig. 6), covering the entire energy range of astrophysical interest. 


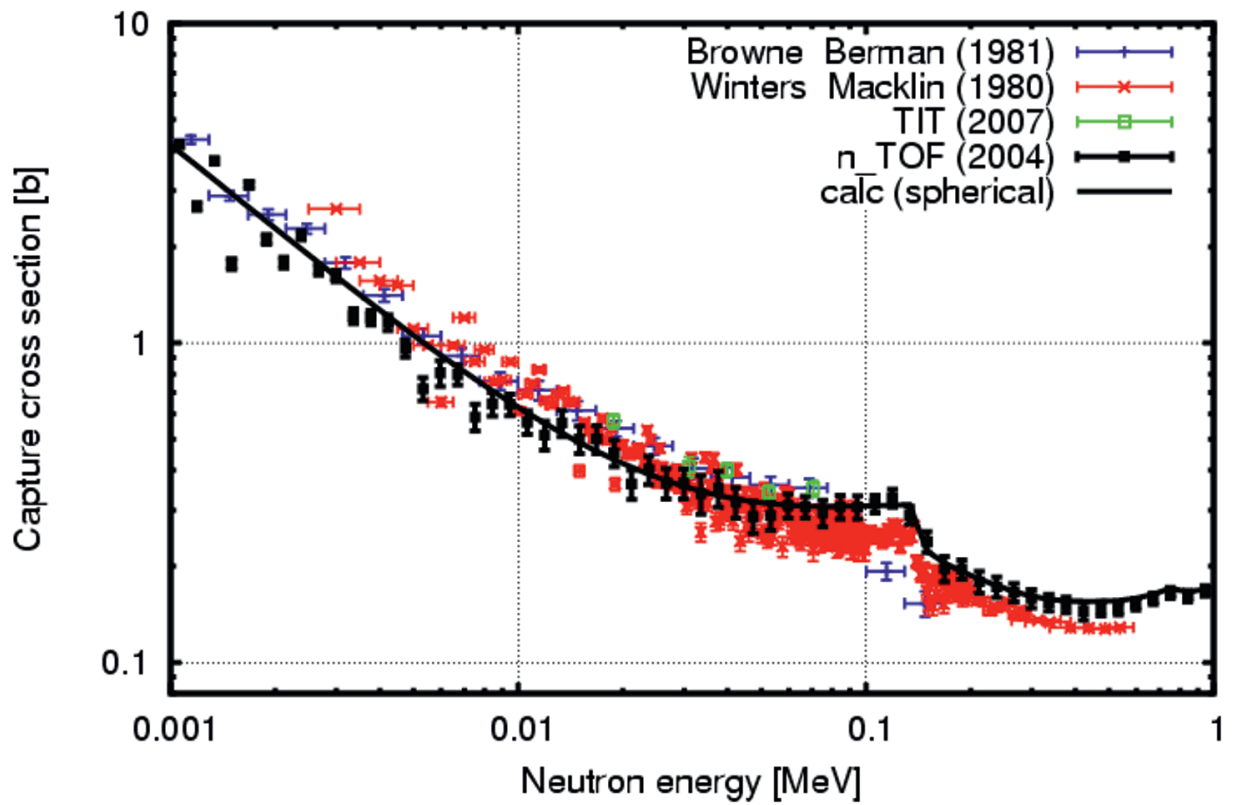

Figure 6. The picture shows the 1870 s (n.g) cross-section measured at the $n$ TOF installation with a set of two C6D6 detectors, compared with previous data.

From these results average level spacings, radiative widths, and neutron strength functions have been deduced by statistical analyses to establish a consistent set of input data for detailed cross section calculations with the Hauser-Feshbach statistical model. Based on these calculations stellar enhancement factors were obtained to correct the Maxwellian averaged cross sections determined from experimental $(n, \gamma)$ data. The corresponding stellar $(n, \gamma)$ cross sections have been used to separate the radiogenic part of the $187 \mathrm{Os}$ abundance from its s-process component and to define the mother/daughter ratio $187 \mathrm{Re} / 187$ Os.

With a schematic model which assumes an exponentially decreasing production rate for $187 \mathrm{Re}$, it was shown that the new data limit the nuclear physics uncertainties for the rhenium-osmium clock to less than $1 \mathrm{Gyr}$, allowing a more accurate estimate for the age of our galaxy: the age of the universe by means of the Re/Os clock is claimed to be $15.3 \pm 0.8 \pm 2 \mathrm{Gyr}$.

\section{FEASIBILITY STUDY FOR EXPERIMENTAL AREA 2 (EAR-2)}

The overall efficiency of the experimental program and the range of possible measurements could be significantly improved with the construction of a 2nd Experimental Area (EAR2), vertically located $20 \mathrm{~m}$ on top of the n_TOF spallation target (see Fig.7) 


\section{EAR-2

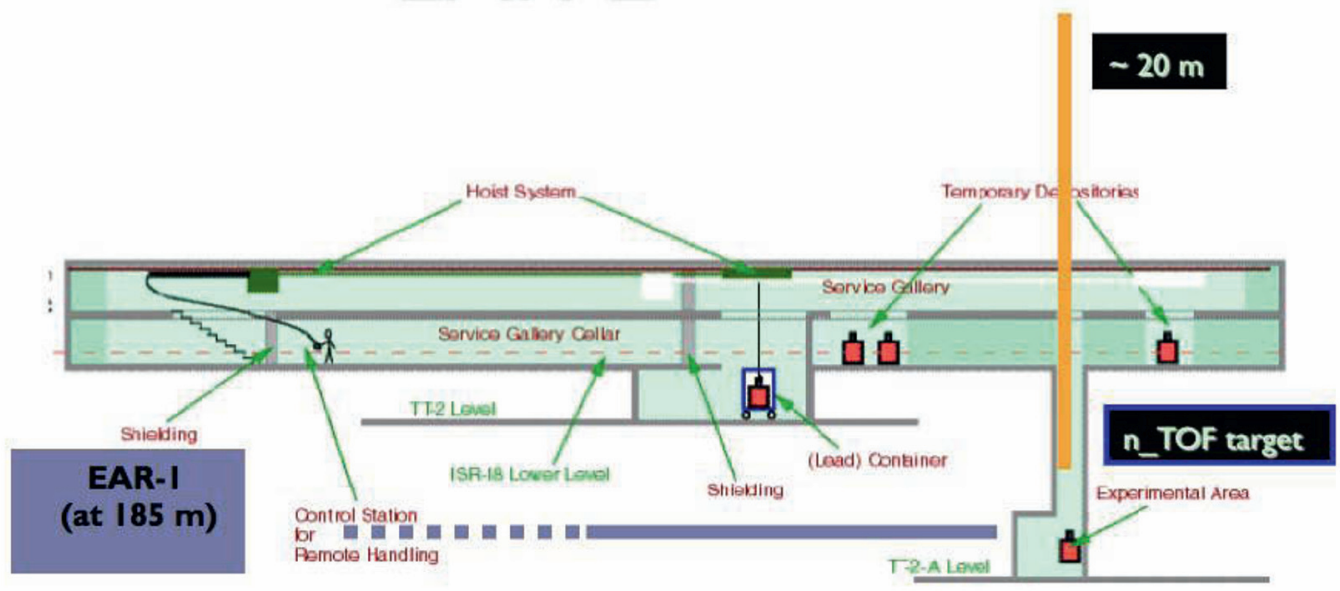

Figure.7: Schematic view of the EAR-1 and of the proposed (EAR-2)

The configuration of the foreseen n_TOF Experimental Area 2 allows to measure neutron-induced reactions with the following advantages:

\subsection{Reduction of $\gamma$-flash.}

Since most of the relativistic particles produced in spallation process and which generate the socalled " $\gamma$ flash" are emitted in the forward direction, placing an experimental area at an angle of 90 degrees with respect to the primary beam axis strongly reduces the related background effects. The strong reduction of these signals, which for some detectors are masking the signal from neutron reaction for the first $\mu \mathrm{s}$, opens the possibility to extend - in those cases - the measurement of neutron induced reactions up to higher energies with respect to what is presently achievable in EAR1.

\subsection{Higher neutron flux}

Being closer to the spallation target (flight path of $20 \mathrm{~m}$ ) the configuration provides a higher instantaneous neutron flux with respect to the present neutron fluence in EAR1 (flight path of $185 \mathrm{~m}$ from the spallation target); this is a clear advantage for the measurement of reactions on samples with very small masses or reactions with very small cross sections. The reduced energy resolution - which is an important aspect for the resolved resonances region - due to the smaller distance between the new experimental area and the target does not affect the measurements at high neutron energies.

The neutron fluence, energy resolution and background are the basic parameters that describe the performances of each facility. Typically each facility made a great effort to reduce the background by introducing appropriate shielding, collimation system etc. However, when short lived radioactive targets are to be measured (as it is the majority of most of the recent $n$ _TOF proposals) the background due to the natural radioactivity can be decreased by decreasing the sample mass, with direct consequence a proportional decrease on the reaction rate $d N_{\text {reaction }} / d t$. The reaction rate 


\section{CNR*11}

is directly proportional to the neutron fluence, which in the case of EAR2 is $\sim 25$ times larger than what is available in EAR1 (see Figure 8).

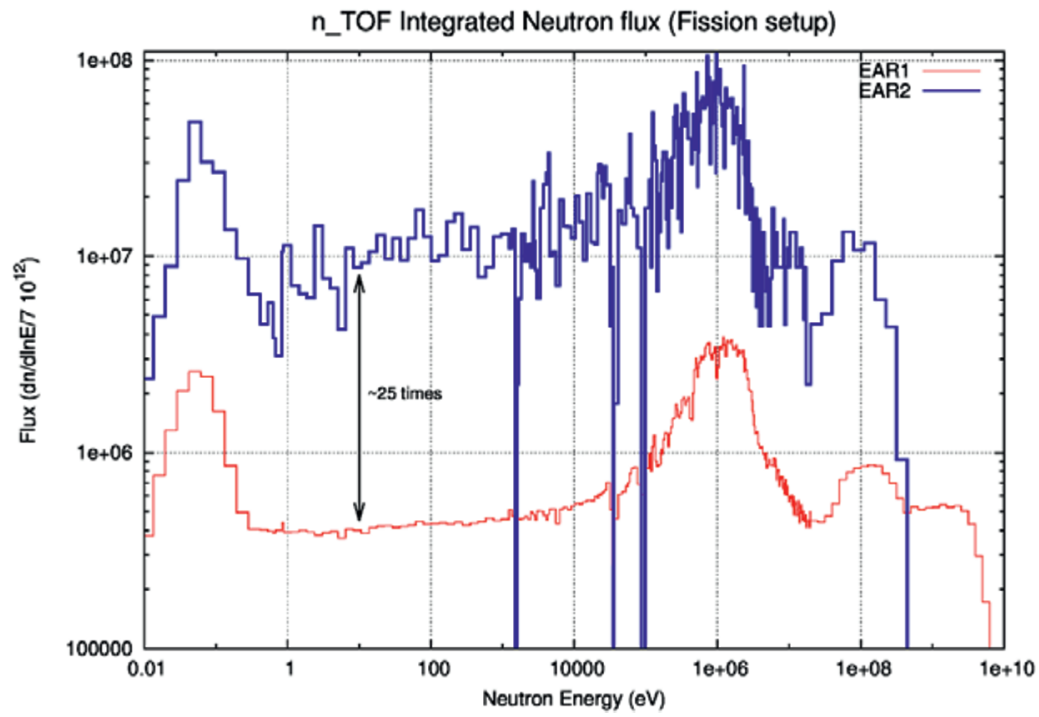

Figure. 8: Comparison between the neutron fluence of the proposed EAR2 and the existing EAR1. The significant absorption dips in the EAR2 fluence are due to the large amount of structural Al between the spallation target and the vertical vacuum tube leading to EAR2.

\subsection{Higher signal to background ratio for radioactive samples}

In the case of time-of-flight measurements on radioactive samples, the background induced by the radioactive decay of the sample is directly proportional to the time needed for the measurement. A range of neutron energy $\Delta E$ corresponds to a window in time-of-flight $\Delta T$, and the signal-to-noise ratio is therefore proportional to the ratio $\Delta E / \Delta T$. From the classical relation between time-of-flight and neutron energy, it follows that $\Delta E / \Delta T$ is inversely proportional to the flight length $L$. Therefore the new short flight path will result in a more favorable signal to radioactive background ratio by a factor $185 / 19$ which equals nearly a factor 10 .

\subsection{Equivalent half-life}

The advantage of the last two points can be combined in comparisons when we define the "equivalent half-life". We would like to compare the rate of neutron-induced reactions

$$
\frac{d N_{\text {reaction }}}{d t}=\sigma(E) \cdot \frac{d n(E)}{d t} \cdot N=\sigma(E) \cdot \frac{2 E}{t(E)} \cdot \Phi(E) \cdot N
$$

with the background from radioactive decays.

$$
\frac{d N_{\text {decay }}}{d t}=\lambda \cdot N=\frac{\log (2)}{t_{1 / 2}} \cdot N
$$

where $d N_{\text {reaction }} / d t$ is the reaction rate, $d N_{\text {decay }} / d t$ the natural decay rate, $t_{1 / 2}$ the isotope half life and $N$ the number of atoms, $\sigma(E)$ the reaction cross section under investigation, $\Phi(E)$ the neutron fluence as a function of the energy, $t(E)$ the time of flight for each neutron. 
From Eq.1 and Eq.2 we can calculate the equivalent half life $t_{1 / 2}(\mathrm{Eq} .3)$ when the reaction rate is equivalent to the decay rate.

$$
t_{1 / 2}=\frac{\log (2)}{\sigma(E) \cdot \frac{2 E}{t(E)} \Phi(E)}
$$

The equivalent half life $t_{1 / 2}$ value only depends on the performances of the facility and the cross section to be measured. This value gives us an indication of the measuring capabilities of the shortest lived isotopes in each experimental area. It is evident that in the EAR2, due to the shorter flight path and the low repetition rate of the PS machine the measuring capabilities are increased further by a factor of 10 on top of the increase of the neutron fluence (factor 25) resulting in a total gain of a factor of about 250 times (with respect to what is achievable presently by performing measurements in EAR1) on measuring radioactive samples with half lives as low as few tenths of years.[fig.9]. Figure 10 shows the equivalent half life in years as a function of the incoming neutron energy, for both measuring stations, the present one EAR1@185m distance, and the future one EAR2@19m distance, and both for two representative values of cross section $\sigma(E)$ of $1 \mathrm{~b}$ and $1 \mathrm{~kb}$. The $1 \mathrm{~b}$ was chosen as a typical value of cross section for higher energies (above $1 \mathrm{MeV}$ ) and the $1 \mathrm{~kb}$ as a typical value for the resonance region ( $1 \mathrm{eV}$ to few $\mathrm{keV})$. Also the half life of some isotopes of the future proposals and past measurements are shown.

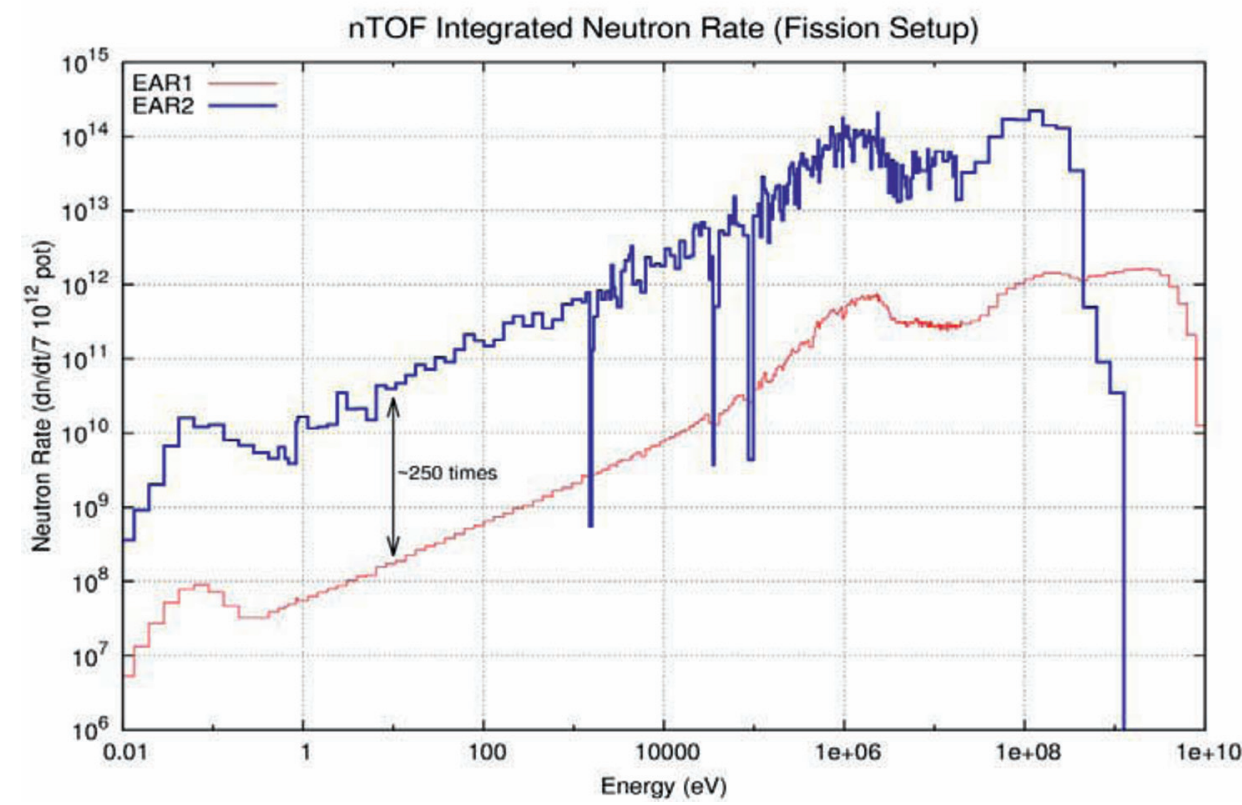

Figure 9. Neutron rate (dn/dt) for the two experimental areas EAR1 and EAR2. 


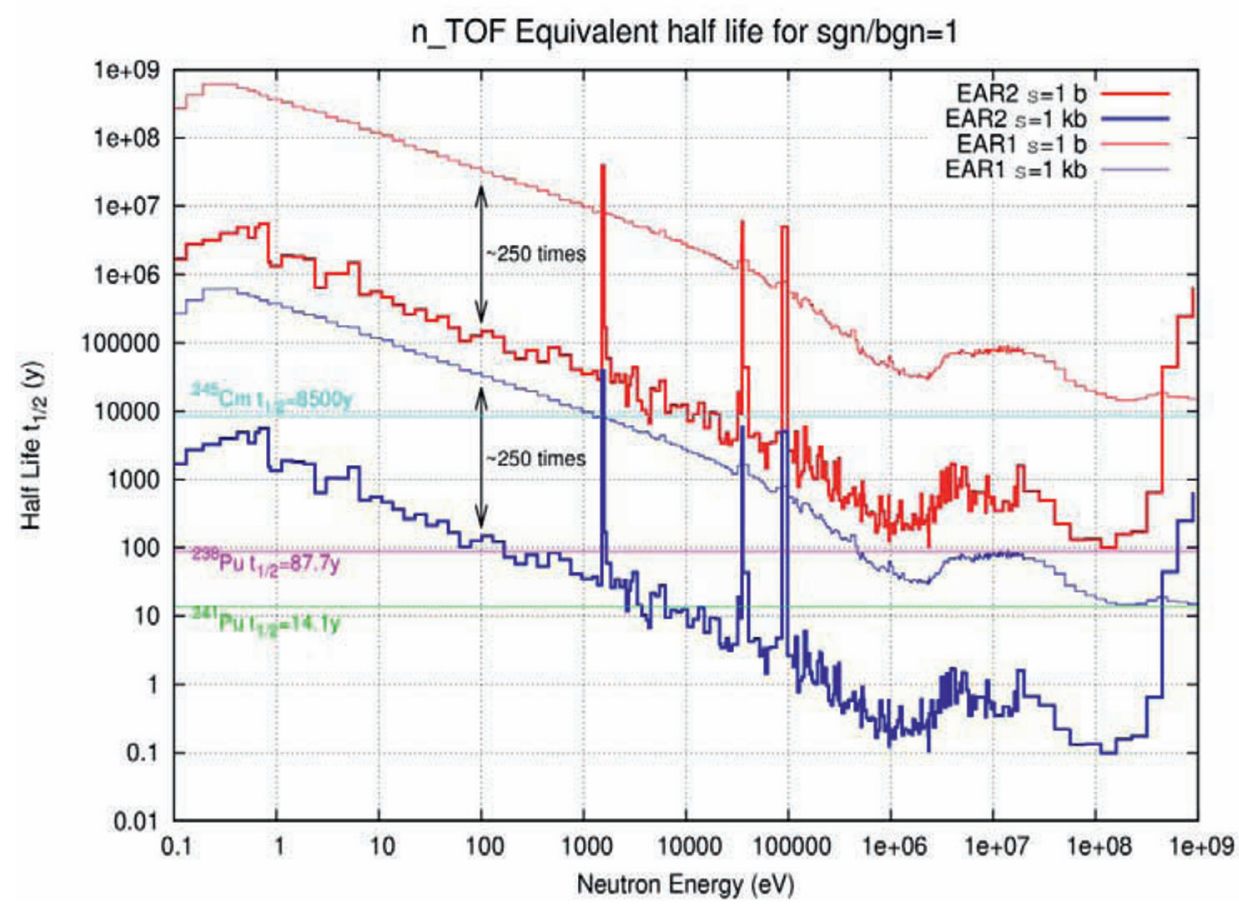

Figure 10. Equivalent half life in years as a function of the incoming neutron energy, for both measuring stations,EAR1@185m andEAR2@19m distance, and both for two representative values of cross section $\sigma(E)$ of $1 \mathrm{~b}$ and $1 \mathrm{~kb}$. Also shown area the half-lives of some proposed isotopes to be measured in EAR2 $\left({ }^{238,241} \mathrm{Pu}\right)$ as well as the one corresponding to ${ }^{245} \mathrm{Cm}$, already measured in EARl.

\section{SCIENTIFIC CASE}

The realization of the $2^{\text {nd }}$ Experimental Area, with its short flight path, will contribute to a substantial improvement in experimental sensitivities and will open a new window to stellar nucleosynthesis, technological issues (such as transmutation or design of safety of future nuclear energy systems) and basic nuclear physics by allowing to measure neutron-induced reactions which are not accessible so far at any other installation.

The main advantages of the $2^{\text {nd }}$ Experimental Area are the following:

- Neutron-induced reaction measurements can be performed on very small mass samples. This feature is crucial to reduce the activity of unstable samples and in cases where the available sample material is limited.(ex. ${ }^{238} \mathrm{Pu},{ }^{241} \mathrm{Pu}{ }^{243} \mathrm{Cm},{ }^{244} \mathrm{Cm},{ }^{245} \mathrm{Cm},{ }^{242} \mathrm{mAm},{ }^{231} \mathrm{~Pa}$, $\left.{ }^{233} \mathrm{~Pa}\right)$

- Measurement can be performed on isotopes with very small cross sections for which the optimization of the signal/background ratio is an essential prerequisite. (ex. ${ }^{86} \mathrm{Kr},{ }^{138} \mathrm{Ba}$, ${ }^{140} \mathrm{Ce},{ }^{208} \mathrm{~Pb}$ )

- Measurement can be performed on much shorter time scales. Repeated runs with modified conditions are essential to check corrections and to reduce systematic uncertainties. 
EPJ Web of Conferences

- Measurements of neutron-induced cross sections at high energies $\left(E_{n}>10-100 \mathrm{MeV}\right)$, which are not possible in the existing EAR-1, will benefit from largely reduced the $\gamma$-flash. This will be particularly important for measurements of ( $n$, charge particle) reactions at high energies because Si and Ge detectors are most strongly affected by the $\gamma$-flash.

\section{CONCLUSION}

CERN n TOF has proven to be a unique facility in the world for its performance.

Since 2011 with its rich scientific program, the n_TOF experiment is contributing to the world efforts aimed at collecting high quality data, mostly on capture and fission neutron-induced reactions.

The transformation of the Experimental Area into Work Sector of Type A has allowed performing measurement of capture and fission cross section of "unsealed" samples of highly radioactive isotopes.

With the construction of the Experimental Area 2, the $n$ _TOF installation is going to open new perspectives in the measurements of fission and capture cross-section with lower samples masses.

\section{REFERENCES}

[1] E.Chiaveri et al. Past,Present and future of the n_TOF facility, Journal of the Korean Physical Society, Vol. 59, No. 2, August 2011, pp. 1620_1623

[2]F.Kaeppeler, Progr.Particles Nucl. Phys. 43(1999) 439

[3]G.Wallestein et al. Rev.Mod.Phys. 69(1997) 995

[4]C.Rubbia et al., A high Resolution Spallation Driven Facility at the CERN-PS to measure Neutron Cross Sections in the Interval from $1 \mathrm{eV}$ to $250 \mathrm{MeV}, \mathrm{CERN} / \mathrm{LHC} / 98-02$ (EET)+Add1

[5]S. Abramovich et al.(the n_TOF Collaboration) European Collaboration for High Resolution Measurements of Neutron Cross Sections between 1eV and 250MeV,CERN/SPSC 99-8,1999

[6]R.Billinge, The CERN PS Complex: A multipurpose Particle Source, Proc.Of XIIth Int. Conf. on High Energy Acc., 1983

[7] U. Abbondanno et al. Neutron capture cross section measurement of $151 \mathrm{sm}$ at the cern neutron time of flight facility (n-tof). Physical review letters, 93(16):161103, 2004.

[8] G. Aerts et al. Neutron capture cross section of th-232 measured at the ntof facility at cern in the unresolved resonance region up to $1 \mathrm{mev}$. Physical Review C, 73(5):054610, 2006.

[9] C. Domingo-Pardo et al. New measurement of neutron capture resonances in bi-209. Physical Review C, 74(2):025807, 2006.

[10] C. Domingo-Pardo et al. Resonance capture cross section of pb-207. Physical Review C, 74(5):055802, 2006.

[11] S. Marrone et al. Measurement of the (151)sm(n, gamma) cross section from $0.6 \mathrm{ev}$ to $1 \mathrm{mev}$ via the neutron time-of-flight technique at the cern n-tof facility. Physical Review C, 73(3):034604, 2006.

[12] C. Domingo-Pardo et al. Measurement of the neutron capture cross section of the s-only isotope pb-204 from 1 ev to $440 \mathrm{kev}$. Physical Review C, 75(1):015806, 2007.

[13] C. Domingo-Pardo et al. Measurement of the radiative neutron capture cross section of 206pb and its astrophysical implications. Physical Review C (Nuclear Physics), 76(4), 2007.

[14] R. Terlizzi et al. The la-139(n,gamma) cross section: Key for the onset of 


\section{$\mathrm{CNR} * 11$}

the s-process. Physical Review C, 75(3):035807, 2007.

[15] G. Tagliente et al. Experimental study of the (91)zr(n, gamma) reaction up to $26 \mathrm{kev}$. Physical Review C, 78(4):045804, 2008.

[16] G. Tagliente et al. Neutron capture cross section of (90)zr: Bottleneck in the s-process reaction flow. Physical Review C, 77(3):035802, 2008.

[17] M. Calviani et al. High-accuracy (233)u(n, f) cross-section measurement at the white-neutron source n-tof from near-thermal to $1 \mathrm{mev}$ neutron energy. Physical Review C, 80(4):044604, 2009.

[18] K. Fujii et al. Neutron physics of the re/os clock. iii. resonance analyses and stellar (n, gamma) cross sections of $(186,187,188)$ os. Physical Review C, 82(1):015804, 2010.

[19] C. Massimi et al. (197)au(n,gamma) cross section in the resonance region. Physical Review C, 81(4):044616, 2010.

[20] C. Paradela et al. Neutron-induced fission cross section of (234)u and (237)np measured at the cern neutron time-of-flight (n-tof) facility. Physical Review C, 82(3):034601, 2010.

[21] G. Tagliente et al. The (92)zr(n,gamma) reaction and its implications for stellar nucleosynthesis. Physical Review C, 81(5):055801, 2010.

[22] F. Belloni et al. Neutron-induced fission cross-section of (233)u in the energy range $0.5 ; \mathrm{e}(\mathrm{n}) ; 20$ mev. European Physical Journal A, 47(1):2, 2011.

[23] C. Lederer et al. (197)au(n,gamma) cross section in the unresolved resonance region. Physical Review C, 83(3):034608, 2011.

[24] G. Tagliente et al. Neutron capture on (94)zr: Resonance parameters and maxwellian-averaged cross sections. Physical Review C, 84(1):015801, 2011.

[25] D. Tarrio et al. Neutron-induced fission cross section of (nat)pb and (209)bi from threshold to $1 \mathrm{gev}$ : An improved parametrization. Physical Review C, 83(4):044620, 2011.

September 26,

[26] M.Calviani et al. Fission Cross Section Measurements of $233 \mathrm{U}, 245 \mathrm{Cm}$ and $241,243 \mathrm{Am}$ at CERN n_TOF facility, Journal of the Korean Physical Society, Vol. 59, No. 2, August 2011, pp. 21062109

[27] D. Cano-Ott et al. Neutron Capture Measurements on Minor Actinides at the $\mathrm{n}$ TOF Facility at CERN: Past, Present and Future, Journal of the Korean Physical Society, Vol. 59, No. 2, August 2011, pp. 1809_1812N 